05

\title{
Анизотропия магнитоэлектрических свойств объемных композитов феррит-пьезоэлектрик
}

\author{
() В.М. Лалетин, ${ }^{1}$ Д.А. Филиппов, ${ }^{2}$ К.И. Янушкевич ${ }^{3}$ \\ ${ }^{1}$ Институт технической акустики НАН Беларуси, \\ 210023 Витебск, Беларусь \\ ${ }^{2}$ Новгородский государственный университет им. Ярослава Мудрого, \\ 173003 Великий Новгород, Россия \\ ${ }^{3}$ НПЦ НАН Беларуси по материаловедению, \\ 220072 Минск, Беларусь \\ e-mail: Dmitry.Filippov@novsu.ru
}

Поступило в Редакцию 19 апреля 2018г.

В окончательной редакции 28 сентября 2018 г.

Принято к публикации 19 ноября 2018 г.

\begin{abstract}
Экспериментально исследована анизотропия магнитоэлектрических свойств объемных композитов на основе цирконата-титаната свинца и феррита-никеля. Показано, что отношение продольного магнитоэлектрического коэффициента к поперечному зависит от величины пьезомодуля композита. Предложен механизм, объясняющий полученные закономерности, в основе которого лежит предположение о том, что анизотропия магнитных свойств обусловлена деформацией, возникающей при электрической поляризации образца.
\end{abstract}

DOI: $10.21883 /$ JTF.2019.05.47467.156-18

\section{Введение}

Магнитоэлектрический (МЭ) эффект проявляется в изменении электрической поляризации вещества при воздействии на него магнитного поля. Если в монокристаллах механизмом МЭ-эффекта является совместное действие спин-орбитального взаимодействия, внутрикристаллического и внешнего электрического поля [1], то в композиционных материалах, состоящих из магнитострикционной и пьезоэлектрической фаз, МЭ-эффект является эффектом вторичного действия или как говорят результатом „product property““ [2]. Изменение электрической поляризации композита происходит в результате механического взаимодействия магнитострикционной и пьезоэлектрической фаз. Механические деформации, возникающие в магнитострикционной фазе при наложении магнитного поля, передаются в пьезоэлектрическую фазу, что вызывает изменение поляризации и приводит к возникновению напряжения на обкладках образца. Существующие МЭ-композиционные материалы можно разделить на слоистые структуры и объемные композиты [3-5]. Объемные композиты изготавливают по керамической технологии путем спекания смесей порошков феррита и пьезоэлектрика. Первоначально такая композиционная керамика является изотропной. После поляризации она приобретает анизотропию, в результате чего такие параметры как пьезомодуль, диэлектрическая проницаемость, модуль Юнга начинают зависеть от ориентации относительно направления поляризации. A priori предполагается, что магнитные свойства композита остаются изотропными. Но процесс поляризации сопровождается деформацией образца. Поэтому вследствие взаимодействия фаз и магнитоупругих эффектов электрическая поляризация композиционной керамики должна привести к появлению анизотропии магнитных свойств. В свою очередь, возникновение магнитной анизотропии должно повлиять на МЭ-характеристики.

Так как величина МЭ-эффекта пропорциональна поляризации, то непосредственные измерения МЭ-коэффициентов не позволяют однозначно судить о наличие магнитной анизотропии. Поэтому в качестве исследуемых параметров использовались коэффициенты $k_{1}$ и $k_{2}$, равные отношению продольного МЭ-сигнала к поперечному для линейного и нелинейного МЭ-эффекта соответственно. Еще одним исследуемым параметром являлась величина напряженности подмагничивающего поля $H_{m}$, при которой наблюдается максимальный линейный МЭ-коэффициент. Цель настоящей работы изучение влияния режимов поляризации образцов, пьезомодуля $d_{33}$ на коэффициенты $k_{1}$ и $k_{2}$ и величину $H_{m}$ поля подмагничивания.

\section{Материалы и методы исследования}

Объемные композиционные МЭ-материалы получены путем спекания смесей порошков феррита и пьезоэлектрика. С этой целью были приготовлены образцы системы $(1-x)$ ЦТС $23-x \mathrm{NiFe}_{1.9} \mathrm{Co}_{0.02} \mathrm{O}_{4}$, где параметр $x$ представляет массовые доли и был равным 0.03, 0.1 и 0.2. Выбор объектов обусловлен достаточно большим содержанием пьезоэлектрической фазы в указанных композитах и, следовательно, можно предположить максимальной деформацией фазы с магнитным упорядочением при электрической поляризации, которая должна привести к возникновению магнитной анизотропии. Образцы спекали в тиглях со свинецсодержащей засыпкой в течение 
$2 \mathrm{~h}$ при температуре $1240^{\circ} \mathrm{C}$. Скорость охлаждения не превышала $50^{\circ} \mathrm{C} / \mathrm{h}$. Электроды наносили путем вжигания серебряной пасты при температуре $650^{\circ} \mathrm{C}$ в течение $20 \mathrm{~min}$. Все образцы имели форму дисков диаметром $8.8 \mathrm{~mm}$ и толщиной $0.87 \mathrm{~mm}$.

Для поляризации образцов использовалось пять режимов: режимы 1-4: поляризация в полях напряженностью $1,2,3,4 \mathrm{kV} / \mathrm{mm}$ при комнатной температуре; режим 5 - поляризация в поле напряженностью $4 \mathrm{kV} / \mathrm{mm}$ при температуре $100^{\circ} \mathrm{C}$.

Перед проведением измерений с целью исключения эффектов, обусловленных долговременной памятью, образцы помещались в переменное размагничивающее поле напряженностью $150 \mathrm{kA} / \mathrm{m}$ и медленно из него извлекались. Образец при этом размагничивался, что обеспечивало хорошую воспроизводимость результатов.

Для нахождения величин коэффициентов $k_{1}$ и $k_{2}$ определялись линейные и нелинейные МЭ-коэффициенты при двух различных ориентациях образца. В одном случае вектор электрической поляризации перпендикулярен постоянному и переменному магнитным полям (поперечный эффект), в другом - параллелен (продольный эффект).

Обычно для исследования МЭ-эффекта в композиционных материалах используется метод, основанный на измерении напряжения, возникающего на образце, при воздействии на него переменного, как правило, с частотой $1 \mathrm{kHz}$, и медленно меняющегося (со скоростью порядка $100 \mathrm{~A} /(\mathrm{m} \cdot \mathrm{s}))$ подмагничивающего поля. Таким образом, находится максимальная величина сигнала и, исходя из толщины образца $(h)$, значения напряжения $(d V)$ и напряженности переменного магнитного поля $(d H)$, определяется МЭ-коэффициент по напряжению $\left(\alpha_{E}\right)$ :

$$
\alpha_{E}=d V /(h d H)
$$

В области переменных магнитных полей $0<d H<1 \mathrm{kA} / \mathrm{m}$ сигнал прямо пропорционален напряженности магнитного поля $d H$, поэтому эффект, исследуемый этим методом, называется линейным МЭ-эффектом. Его величина пропорциональна пьезомагнитному коэффициенту.

Существует также другой метод, применяемый для исследования МЭ-эффекта. Он основан на измерении напряжения $V$, возникающего на образце, при воздействии на него переменного магнитного поля с медленно меняющейся амплитудой. В данном случае МЭ-коэффициент имеет размерность напряженности электрического поля. Обычно представляет интерес величина коэффициента $E_{m}$, определяемая в режиме насыщения сигнала:

$$
E_{m}=V_{m} / h
$$

Он позволяет определить максимальную напряженность электрического поля, которая генерируется в данном материале под действием магнитного поля. Его значение пропорционально величине магнитострикции. В литературе эта характеристика получила название нелинейного МЭ-эффекта [6]. Необходимо отметить, что деление на линейный и нелинейный МЭ-эффекты носит чисто условный характер. Оба метода предназначены для исследования МЭ-эффекта. Первый метод позволяет получить дифференциальную характеристику МЭ-эффекта (линейный МЭ-эффект), второй - интегральную характеристику МЭ-эффекта (нелинейный МЭ-эффект). Оба метода и соответственно линейный и нелинейный МЭ-эффекты связаны между собой [6]. Знание полевой зависимости одного МЭ-коэффициента позволяет путем интегрирования или дифференцирования найти полевую зависимость второго МЭкоэффициента $[7,8]$.

\section{Результаты исследования и их обсуждение}

Максимальные значения МЭ-коэффициентов при линейном и нелинейном эффектах получены на образце, содержащем 20 mass. \% феррита никеля, поляризованным при $100^{\circ} \mathrm{C}$. На рис. 1 показана зависимость продольного и поперечного линейного МЭ-коэффициента от подмагничивающего поля. Фазы МЭ-сигналов отличаются на 180 градусов. Это обусловлено различными знаками продольной и поперечной магнитострикций. Величина коэффициента при продольном эффекте составляет $131.3 \mathrm{mV} / \mathrm{A}$ при подмагничивающем поле $74 \mathrm{kA} / \mathrm{m}$. Для поперечного эффекта коэффициент достигает максимальной величины $78.3 \mathrm{mV} / \mathrm{A} \mathrm{в} \mathrm{магнитном}$ поле $31 \mathrm{kA} / \mathrm{m}$. На рис. 1 представлено проявление гистерезиса в интервале магнитных полей $0<H<100 \mathrm{kA} / \mathrm{m}$. При поперечном эффекте величина коэрцитивной силы равна $3.5 \mathrm{kA} / \mathrm{m}$, при продольном эффекте - в два раза меньше $1.8 \mathrm{kA} / \mathrm{m}$. Полученный результат еще раз подчеркивает, что в основе возникновения МЭ-эффекта лежат перекрестные эффекты, обусловленные упругим взаимодействием фаз. Исходя из логики явлений намагничивания, величина коэрцитивной силы при продольном эффекте должна быть больше значения коэрцитивной силы при поперечном эффекте вследствие влияния размагничивающих полей.

На рис. 2 приведены полевые зависимости нелинейного МЭ-ффекта при продольной и поперечной ориентации поляризации образца относительно магнитного поля. В случае поперечного эффекта МЭ-сигнал выходит на насыщение в полях свыше $100 \mathrm{kA} / \mathrm{m}$ составляет $4.9 \mathrm{~V} / \mathrm{mm}$. При продольном эффекте МЭ-сигнал достигает величины $15.5 \mathrm{~V} / \mathrm{mm}$ и выходит на насыщение в полях свыше $200 \mathrm{kA} / \mathrm{m}$. Следует отметить, что вследствие нелинейности в режиме насыщения наряду с откликом на основной частоте возникает отклик на удвоенной и т.д. частотах $[9,10]$. Однако данные отклики хорошо проявляются либо при отсутствии поля подмагничивания, когда отклик на основной частоте отсутствует, либо при слабых полях, когда его величина мала. В области насыщения величина отклика на основной гармонике 


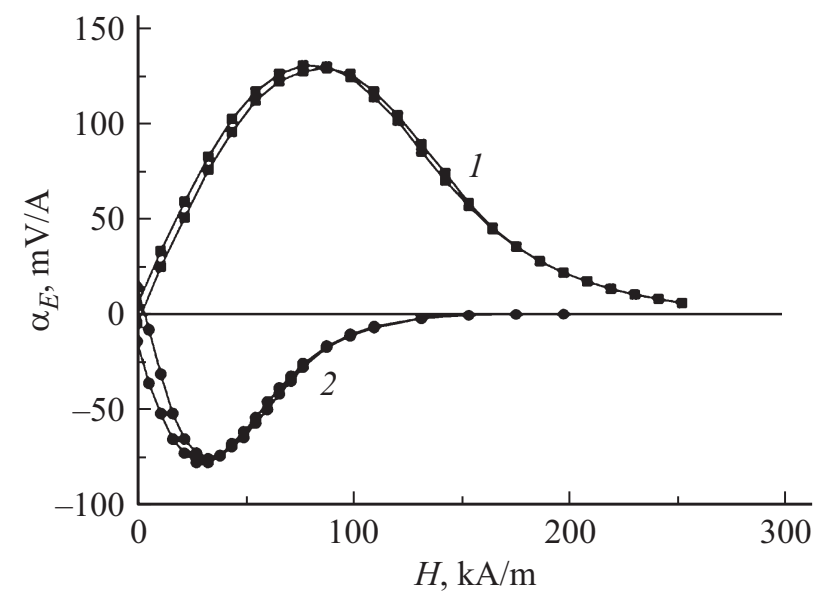

Рис. 1. Полевая зависимость продольного (1) и поперечного (2) линейного МЭ-коэффициента для образца состава 3, поляризованного при $100^{\circ} \mathrm{C}$.

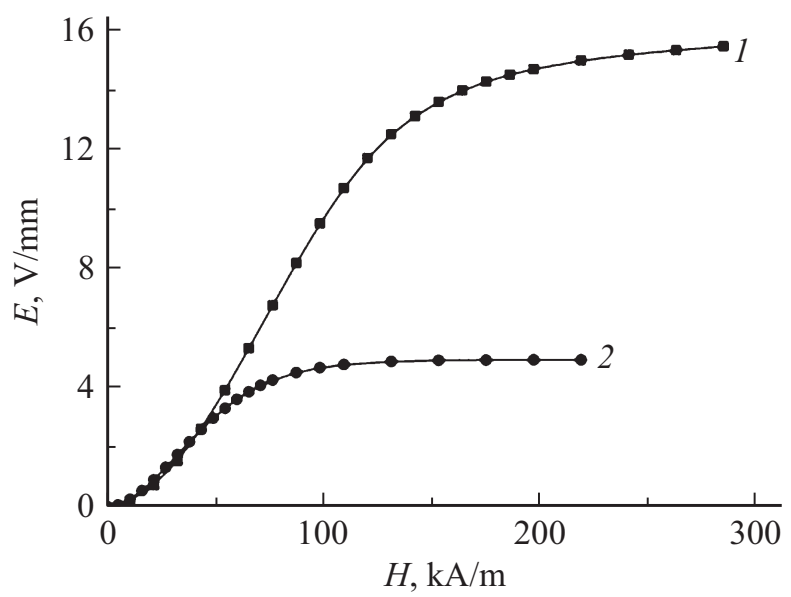

Рис. 2. Полевая зависимость продольного (1) и поперечного (2) нелинейного МЭ-коэффициента для образца состава 3, поляризованного при $100^{\circ} \mathrm{C}$.

значительно превосходит величину отклика на высших гармониках и последними в первом приближении можно пренебречь.

Полевые зависимости МЭ-коэффициентов, полученные для образцов других составов, имеют аналогичный вид, отличающийся величиной сигнала и значениями магнитных полей.

Изучение влияния условий поляризации композиционной керамики на величину поля подмагничивания $H_{m}$ показало, что с увеличением пьезомодуля существует устойчивая тенденция уменьшения напряженности поля $H_{m}$ при поперечном эффекте и ее увеличение при продольном эффекте (рис. 3). Для составов, содержащих 3, 10 и 20 mass.\% феррита никеля, абсолютное изменение величины $H_{m}$ при поперечном эффекте составило 5.5, $4.6,2.8 \mathrm{kA} / \mathrm{m}$, а при продольном эффекте - 8.3, 5.5, $3.9 \mathrm{kA} / \mathrm{m}$. Из результатов эксперимента следует, что максимальные изменения величины $H_{m}$ имеют место для состава с содержанием феррита 3 mass.\%. С увеличением содержания магнитной фазы в композите уменьшается влияние поляризации на изменение величины напряженности поля подмагничивания.

Результаты изучения влияния величины пьезомодуля на коэффициенты $k_{1}$ и $k_{2}$ при линейном и нелинейном эффектах представлены на рис. 4 и 5. Установлено, что с увеличением пьезомодуля увеличиваются коэффициенты $k_{1}$ и $k_{2}$. Для линейного эффекта значения коэффициента $k_{1}$ изменяются от 1.15 до 2.56 , от 1.10 до 2.16 и от 1.11 до 1.68 для образцов, содержащих 3, 10 и 20 mass.\% феррита соответственно. Полученные зависимости являются практически линейными и расположены на одной прямой. Коэффициент $k_{1}$ отражает отношение пьезомагнитных коэффициентов. На их величину влияет состав керамики и форма исследуемых образцов.

В случае нелинейного эффекта наблюдается экспоненциальная зависимость коэффициента $k_{2}$ от величины пьезомодуля. Для составов, содержащих 3, 10 и 20 mas.\%

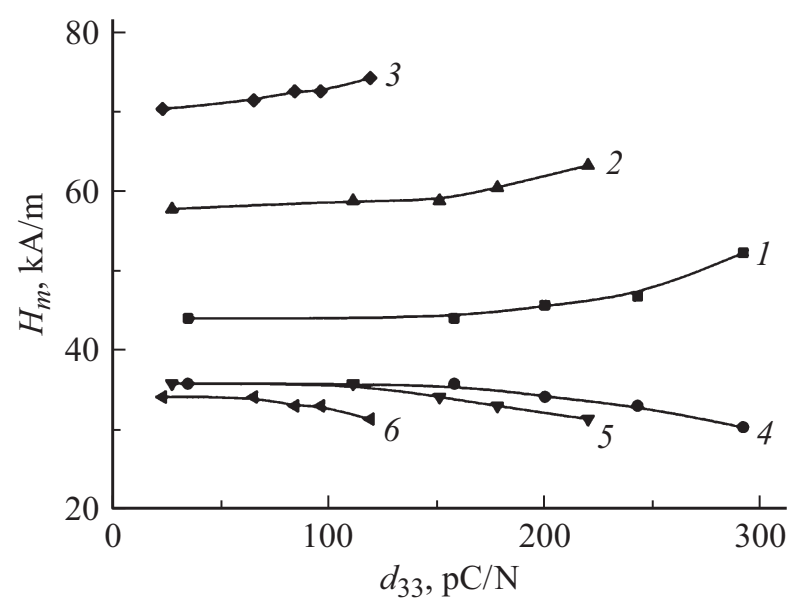

Рис. 3. Зависимость величины подмагничивающего поля продольного (1-3) и поперечного (4-6) МЭ-эффекта от пьезомодуля для образцов составов $1-3$.

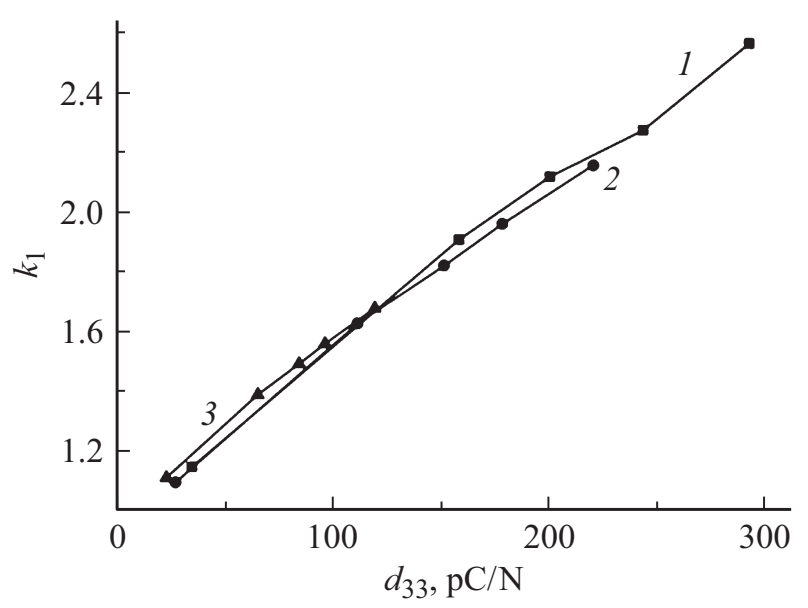

Рис. 4. Зависимость коэффициента $k_{1}$ от пьезомодуля при линейном эффекте для образцов, содержащих 3 (1), 10 (2) и 20 mass.\% (3) феррита. 


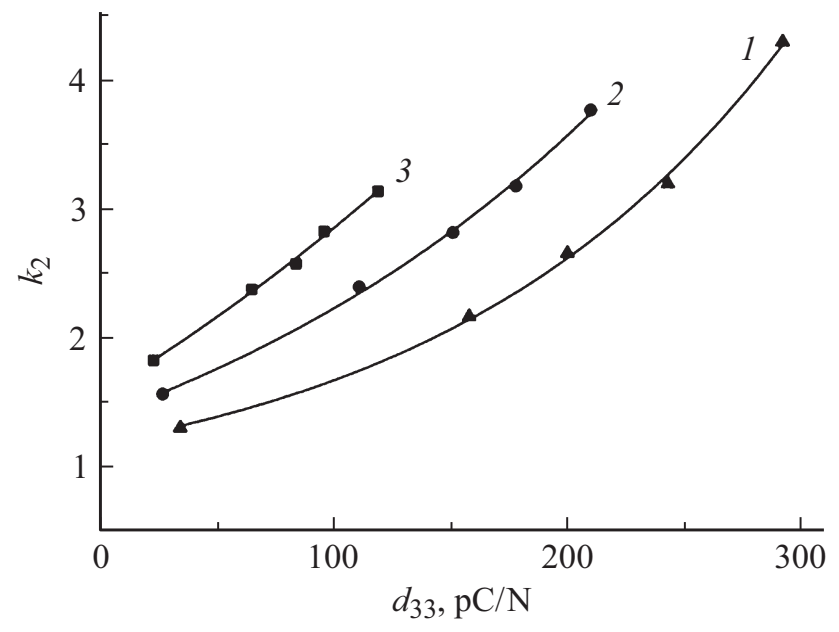

Рис. 5. Зависимость коэффициента $k_{2}$ от пьезомодуля при нелинейном эффекте для образцов, содержащих 3 (1), 10 (2) и 20 mass.\% (3) феррита.

феррита, коэффициент меняется соответственно от 1.31 до 4.16, от 1.57 до 3.77 и от 1.83 до 3.14. Экстраполяция полученных зависимостей до величины пьезомодуля, равного нулю, позволяет получить значения коэффициента $k_{2}$ для неполяризованного состояния композиционной керамики. Составы, содержащие 3, 10 и 20 mass.\% феррита, имеют значения коэффициента $k_{2}$, равные соответственно $1.09,1.40$ и 1.62 . Известно, что отношение продольной магнитострикции к поперечной для феррита никеля равно 2.0 [11]. Принимая во внимание, что коэффициент $k_{2}$ отражает отношение продольной магнитострикции к поперечной, можно предположить, что магнитная фаза находится в композите в „сжатом“ состоянии. В неполяризованной изотропной керамике $\mathrm{c}$ уменьшением содержания пьезоэлектрической фазы ее влияние на магнитную фазу уменьшается. В результате коэффициент $k_{2}$ увеличивается от 1.09 до 1.62 . Электрическая поляризация снимает механические напряжения в направлении поляризации и усиливает их в перпендикулярном направлении. Это приводит к возникновению магнитной анизотропии и, как следствие, к увеличению коэффициентов $k_{1}$ и $k_{2}$.

\section{Заключение}

Установлено, что в объемных композиционных материалах $(1-x)$ ЦТС23 $-x \mathrm{NiFe}_{1.9} \mathrm{Co}_{0.02} \mathrm{O}_{4}$, где $\mathrm{x}=0.03,0.1,0.2$, электрическая поляризация сопровождается возникновением анизотропии в магнитной фазе. Это приводит к изменению величины напряженности $H_{m}$ поля подмагничивания, при которой наблюдается максимальный линейный МЭ-коэффициент, а также к зависимости коэффициентов $k_{1}$ и $k_{2}$, равных отношению продольного МЭ-сигнала к поперечному для линейного и нелинейного МЭ-эффекта, от величины пьезомодуля исследуемых керамических составов.
Работа выполнена при поддержке гранта совместного конкурса РФФИ - БРФФИ: Российский проект № 18-52-00021 и Белорусский проект № Ф18Р-300.

\section{Список литературы}

[1] Bichurin M.I., Filippov D.A. // Ferroelectrics. 1997. Vol. 204. $\mathrm{N}$ 14. P. 225-232.

[2] Van Suchtelen // Philips Res. Rep. 1972. Vol. 27. P. $28-37$.

[3] Ryu J., Carazo A.V., Uchino K., Kim H. // J. Electroceramics. 2001. Vol. 7. P. 17-24.

[4] Boomgaard Van Den J., Born R.A.J. // J. Mater. Sci. 1978. Vol. 13. N 5. P. $1538-1548$.

[5] Филиппов Д.А., Лалетин В.М. // ЖТФ. 2012. Т. 82. Вып. 1. C. 47-51.

[6] Лалетин В.М., Поддубная Н.Н. // Письма в ЖТФ. 2017. Т. 43. Вып. 2. С. 60-67.

[7] Laletin V.M., Petrov V.M. // SSC. 2011. Vol. 151. N 23. P. 1806-1809.

[8] Boomgard Van Den J., Run Van A.M.J.G., Suchtelen Van J. // Ferroelectrics. 1976. Vol. 10. P. 295-298.

[9] Burdin D., Chashin D., Ekonomov N., Fetisov L., Fetisov Y., Shamonin M. // J. Phys. D: Appl. Phys. 2016. Vol. 49. P. 375002.

[10] Филиппов Д.А., Лалетин В.М., Фирсова Т.О. // ФТТ. 2014. Т. 56. Вып. 5. С. 944-948.

[11] Аннаев Р.Г., Оразсахатов А. В сб.: Физические и физикохимические свойства ферритов / Под ред. Н.Н. Сироты. Минск: Наука и техника, 1966. С. 155. 